\title{
The Surgical Management of the Rare Neurogenic Myositis Ossificans of the Hip: A Report of 3 Cases
}

\author{
Yazan Hammad ${ }^{1}$, Reem Akiely ${ }^{2}$, Nada Hajjaj ${ }^{2}$, Farah Tahboub ${ }^{2}$,jihad Al-Ajlouni ${ }^{1}$ \\ Learning Point of the Article: \\ Careful radiographic and metabolic evaluation are needed in the $\mathrm{MO}$ before the surgical intervention
}

\section{Abstract}

Introduction: Myositis ossificans (MO) is a benign non-neoplastic condition in which heterotopic bone formation occurs in soft tissues. Neurogenic $\mathrm{MO}$ is one variant of $\mathrm{MO}$ where the lesion is a result of neurological disorders, including brain and spinal cord injuries, especially when followed by immobility and spasticity. MO can also be a result of direct trauma or even genetic mutations.

Case Report: We present three cases of young men (16,37, and 22-year-old) who developed MO of the hip joint following brain or spinal cord injuries. One of them had also sustained a direct trauma to the affected hip joint at the time of the accident. All three patients presented with inability to walk independently due to diminished range of motion at the affected joint. X-rays and computerized tomography (CT) scans with 3dimentional (3D) reconstruction suggested the diagnosis of $\mathrm{MO}$, but the serum alkaline phosphatase was within normal limits at the time of presentation. The first case had bilateral involvement with unmistakable separation between the heterotopic bone formation and the frank hip joints on CT. This patient underwent successful staged excision of the ossifications. The second patient had unilateral hip joint involvement with the absence of clear separation between the heterotopic bone formation and the hip joint, thus, underwent total hip replacement for the affected side as excision was not possible. The third patienthad unilateral hip joint involvement and underwent excision of the ossification with dynamic hip screw insertion after sustaining a stable intertrochanteric fracture intraoperatively. Postoperatively, all three patients received physiotherapy and oral indomethacin. Upon recovery, they were able to walk independently with a near-normal range of motion at the hip joint. There was no evidence of recurrence upon follow-up visits, and CT scans in patients I and II. Follow-ups for patient III were not possible as the patient died 1 month after surgery due to pulmonary embolism.

Conclusion: The surgical management of $\mathrm{MO}$ is indicated when non-operative methods fail to provide an adequate range of motion around the hip joint. Pre-operative assessment utilizing 3D-CT scans proved to be essential in dictating the appropriate surgical approach. During postoperative follow-ups, the physiotherapy and oral indomethacin provided additional improvement in outcome and patients' satisfaction.

Keywords: Myositis ossificans, total hip replacement, arthrolysis.

\section{Introduction}

Myositis ossificans (MO), often referred to as heterotopic ossification ( $\mathrm{HO})$, is a rare benign non-neoplastic condition in which heterotopic bone formation occurs in soft tissues, with muscles being mostly involved $[1,2,3]$. One variant of $\mathrm{MO}$, termed $\mathrm{MO}$ circumscriptaor traumatica, can occur as a result of a direct blow or repeated minor trauma and is estimated to account for the majority of cases of $\mathrm{MO}[1,4]$. MO, without a history of direct trauma to the involved region, can be seen with burns, hemophilia, and neurological disorders [1]. Prolonged immobility and spasticity increase the risk of developing neurogenic $\mathrm{HO}$ following traumatic brain or spinal cord injuries[5]. A genetic variant of $\mathrm{MO}$, termed $\mathrm{MO}$ progressiva, is associated with the condition fibrodysplasia ossificans progressiva, a rare autosomal dominant disorder caused by a mutation in AVCR1 gene resulting in congenital skeletal

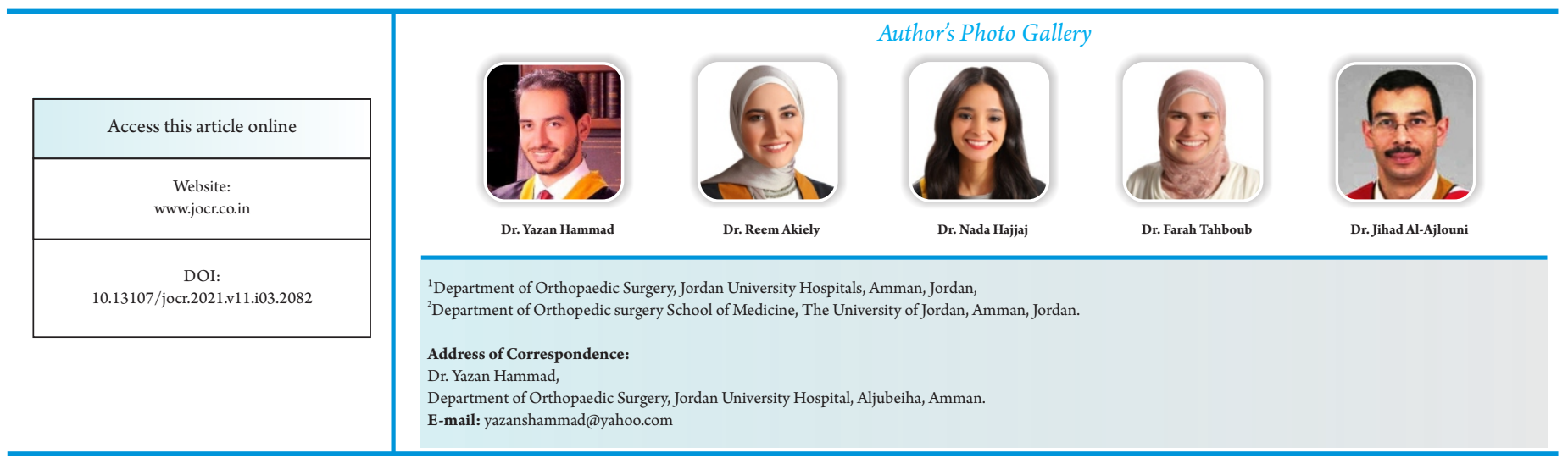

Journal of Orthopaedic Case Reports | pISSN 2250-0685 | eISSN 2321-3817 | Available on www.jocr.co.in | doi:10.13107/jocr.2021.v11.i03.2082 This is an Open Access article distributed under the terms of the Creative Commons Attribution Non-Commercial License (http://creativecommons.org/licenses/by-nc/3.0) which permits unrestricted non-commercial use, distribution, and reproduction in any medium, provided the original work is properly cited. 


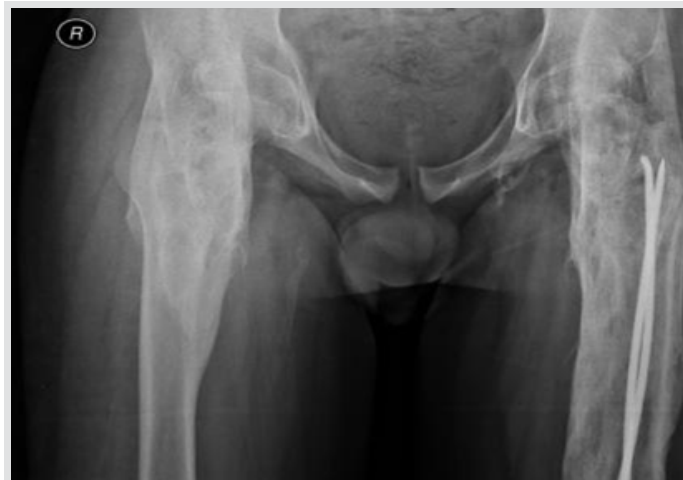

Figure 1: Case I, a radiograph at presentation, with bilateral adduction and ankylosis of the hip.

malformations and progressive $\mathrm{HOs}$ [6].

$\mathrm{MO}$ can present with a variety of clinical symptoms, ranging from minor swelling to severe pain and joint stiffness [7]. For the diagnosis, radiographic findings are often sufficient; however, computerized tomography (CT) scanning, magnetic resonance imaging (MRI), or bone scintigraphy may be necessary in certain instances such as in early disease process; before any calcifications have settled, or when another soft tissue pathology is suspected $[7,8,9,10,11]$. Moreover, it is important to acknowledge the important role that biopsy plays in the diagnostic process of $\mathrm{MO}$, specifically, whenever any indeterminate lesion is encountered and imaging is non-diagnostic [7]. Given the benign and selflimiting natural course of the disease process, conservative nonsurgical management focusing on symptomatology control is often the preferred choice when it comes to the management of MO [12]. Surgical excision is generally reserved for lesions that fail to be controlled with conservative management and/or introduce severe limitations to the activities of daily living [ 7 , 13]. In this report, we present three cases of MO of the hip joint from Jordan University Hospital that was managed by the Orthopedic Surgery Department between the years 2016 and 2020.

\section{Case Report}

\section{Case I}

A 16-year-old boy who was involved in a quarrel sustained a stab wound in the back at the level of D10 that resulted in internal bleeding and vascular injury to the spinal cord with immediate paraplegia and urinary incontinence. He received physical therapy that led to partial recovery. The patient regained continence and became able to move from bed to wheelchair. During one physiotherapy session, the patient sustained a left femur shaft fracture and was treated by flexible nails. He presented to us 1 year after the initial injury with severe bilateral hip ankylosis (Fig. 1).On physical examination, the patient could move from bed to chair and walk only a few steps with flexed hips and adduction, neutral rotation, and extension of both lower limbs. Power was $4 / 5$ all over the lower extremities bilaterally, with hypoesthesia below D10 level (i.e., the level of stab injury). The rest of the physical examination was unremarkable.

Radiographs of the hip joints revealed evident ossification that suggested the diagnosis of MO(Fig. 1). Moreover, 3-dimentional (3D)-CT scan(Fig. 2)revealed anterior and posterior ossification around the hip joint bilaterally. Importantly, however, the $\mathrm{HO}$ was separated from the actual femur bone and hip joint by soft tissue. Serum alkaline phosphatase (SAP) level was within normal limits and bone scan was normal. The patient underwent successful staged excision of the ossifications; the right side followed by the leftside after 1 month (Fig. 3). Upon recovery from the operation, the patient was able to walk independently with residual hip flexion. Postoperatively, he received physiotherapy and indomethacin ( $75 \mathrm{mg}$ PO for 6 weeks) to prevent a recurrence. Follow-ups over 5 years demonstrated achievement of community ambulation without assistance and no signs of recurrence of $\mathrm{MO}$ (Fig.4).

\section{Case II}

A 37-year-old man who was involved in a car accident sustained a head injury and a left acetabular fracture. The head injury yielded intracerebral hemorrhage and the patient was in a coma for several months. After regaining his full consciousness, he was bed-bound with the inability to walk independently or mobilize from bed to chair, with evident left-sided hemiparesis. The patient received physical therapy that led to restoring his ability to walk only with the aid of a walker. He presented to us 1.5 years

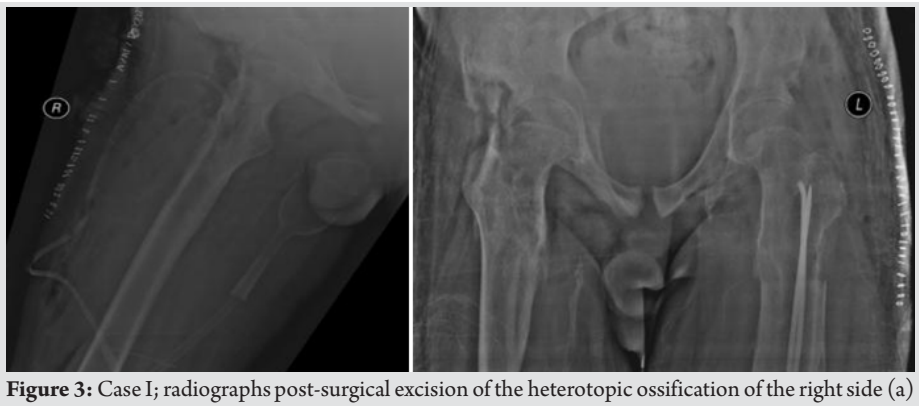
Figure 3: Case I; radiographs post-surgical excision of the heterotopic ossification of the right side (a) followed bythe left side (b) 

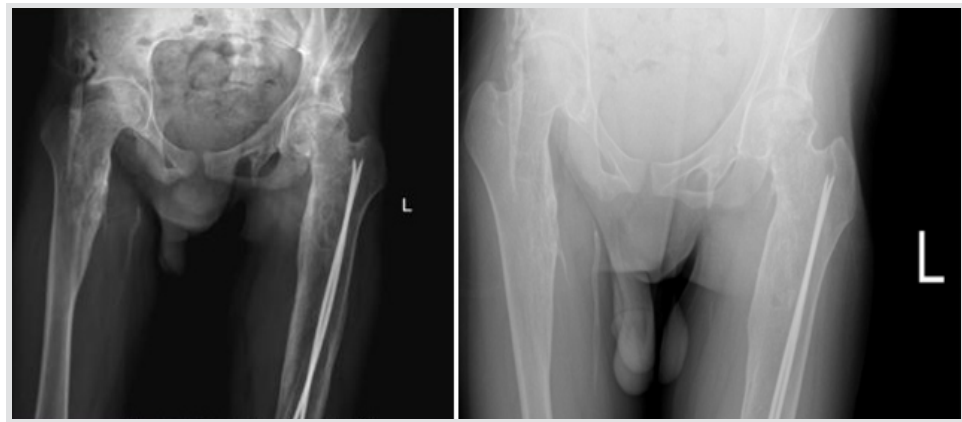

Figure 4: Case I;radiographs lyear (a) and 5 years (b) post-operative showed no recurrence of myositi ossificans.

after the initial accident with severe left hip ankylosis(Fig. 5). On physical examination, the patient was continent; the left hip joint was absolutely stiff with flexion deformity. The right hip had a completely normal range of motion. The rest of the physical examination was unremarkable.

Radiographs of the hip joints revealed evident ossification(Fig. 5) and 3D-CT(Fig. 6) revealed anterior and posterior ossification around the left hip joint that suggested the diagnosis of MO. No soft tissue was visualized between the ossification and the actual femur bone around the hip. At the presentation, the level of SAP was within normal limits. The patient was consented for arthrolysis versus left total hip replacement but eventually underwent total hip replacement as excision was not possible intraoperatively (Fig. 7). Upon recovery from the operation, the patient was able to walk independently with residual hip flexion. Postoperatively, the patient received physiotherapy and indomethacin ( $75 \mathrm{mg}$ PO for 6 weeks) to prevent a recurrence. Follow-ups over 4 years demonstrated the full function of the affected limb and no signs of recurrence (Fig. 8).

\section{Case III}

A 22-year-old man who was involved in a car accident sustained a hemorrhagic cerebrovascular accident. His hospital stay extended for several months and was remarkable for prolonged intubation (2.5 months), tracheostomy tube placement, jejunostomy tube placement, and recurrent chest infections. During his hospital stay, the patient did not receive any lower limb
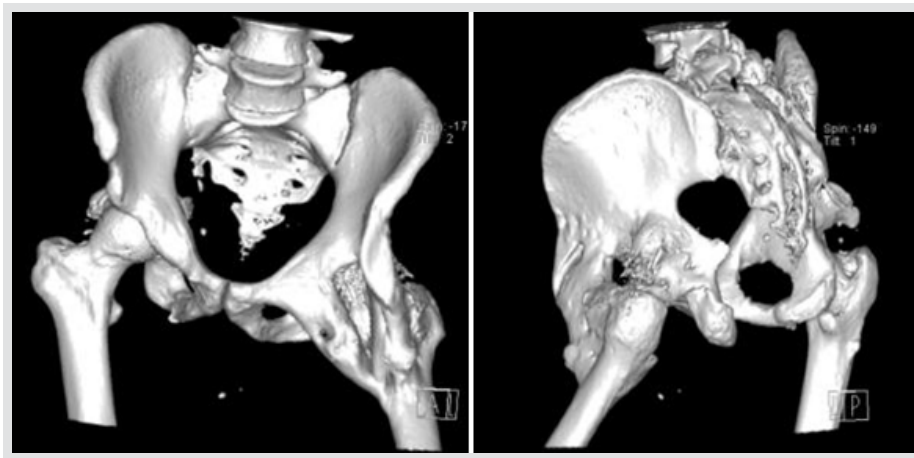

Figure 6: Case II; computerized tomography-3D at presentation revealing left hip ankylosis.

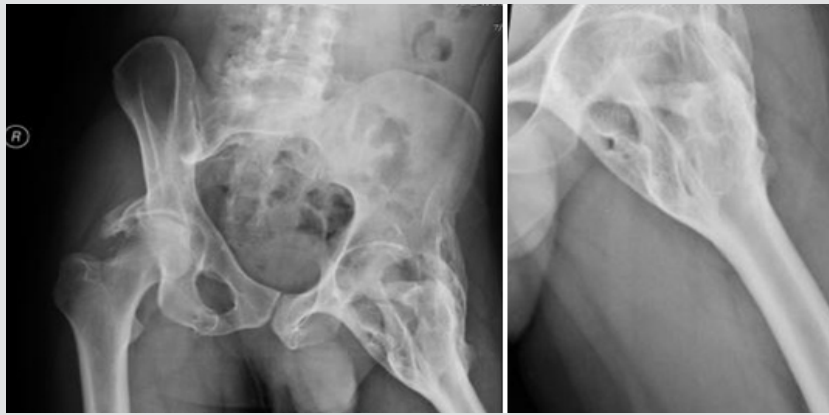

Figure 5: Case II; radiographs at presentation revealingleft hip ankylosis.

physical therapy. Upon discharge, he had right-sided spastic hemiparesis with aphasia and was started on private physiotherapy and rehabilitation. He presented to our outpatient clinic after 1.5 years of physiotherapy with significant resistance and progressive limitation in the range of movement around the right hip joint. At this stage, the patient was bed ridden with the inability to ambulate independently. On physical examination, the patient was continent, the right hip joint was absolutely stiff with flexion deformity and fixed external rotation, and power was $4 / 5$ all over the right lower extremity with intact sensation. His left hip joint had a completely normal range of motion and the rest of the physical examination was unremarkable.

Radiographs of the right hip joint revealed evident ossification(Fig. 9). On 3D-CT, HO surrounding the right hip joint was seen associated with atrophied muscles and partial fusion of the proximal femur and dorsal acetabulum/ischium, causing fixed external rotation of the right femur (Fig. 10). SAP level at presentation was within normal limit. The bone scan initially demonstrated increased blood flow and hyperemia at the involved region, suggesting that the observed $\mathrm{HO}$ was immature; however, when repeated after 6 months, the bone scan was normal. The patient was consented for excision and arthrolysis. Intraoperatively, the patient sustained a stable intertrochanteric fracture and a dynamic hip screw (DHS) was inserted (Fig. 11).Postoperatively, there was a significant improvement in the range of motion with physiotherapy and oral indomethacin as the first two cases. Sadly, however, the follow-up was only for 1 month as the patient died from a pulmonary embolism.

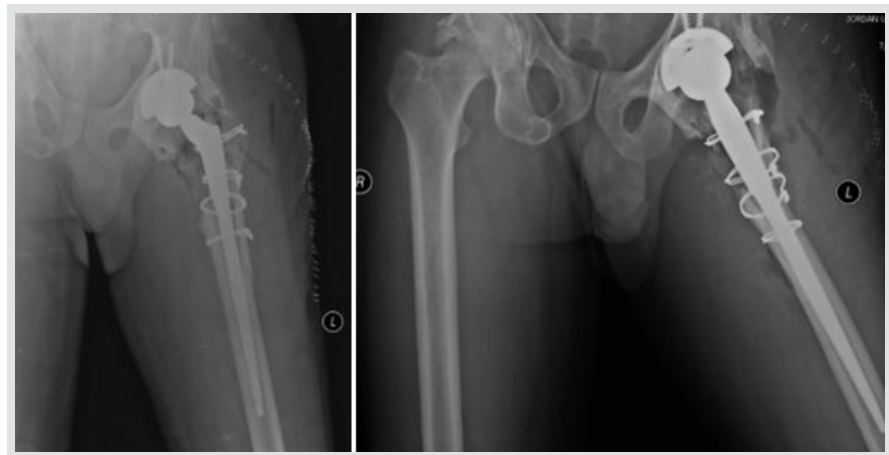

Figure 7: Case II;radiographs post excision of heterotopic ossification and left total hip replacement. 


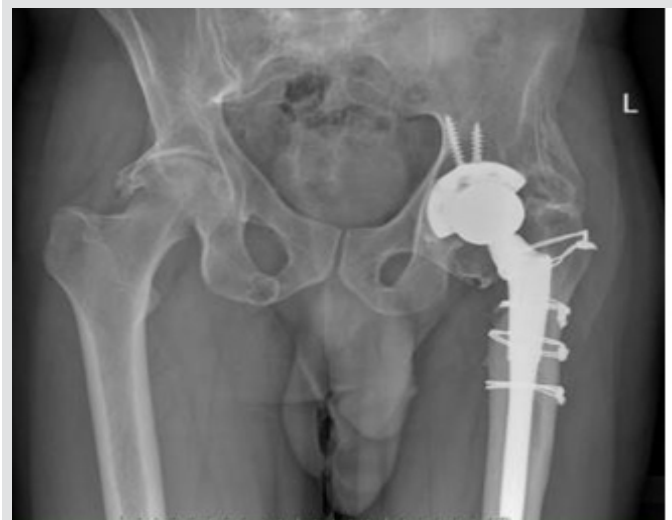

Figure 8: Case II; radiographs at 4-yearfollow-up demonstrated no recurrence.

\section{Discussion}

$\mathrm{MO}$, often referred to as $\mathrm{HO}$, is a rare benign non-neoplastic condition in which heterotopic bone formation occurs in soft tissues, with muscles being mostly involved $[1,2,3]$. Even though $\mathrm{MO}$ is relatively an uncommon condition, it has been well described in the literature possessing distinct clinical, radiological, and pathological properties [7]. It is suggested that the pathogenesis of $\mathrm{MO}$ formation is dependent on dysregulation of local stem cells in response to injury-induced inflammation with resultant endothelial-mesenchymal transition; a process in which certain cytokines released as part of the inflammatory cascade drive endothelial cells of skeletal muscles to undergo endothelial-mesenchymal transition [14, 15]. These endothelial-derived mesenchymal stem cells are described as the key players in the pathogenesis of $\mathrm{MO}$, as they are the source of heterotopic bone formation in extra-skeletal tissues [15]. Conventionally, MO was linked to antecedent trauma, especially sport-related injuries. However, the attention toward non-traumatic cases has grown over the past few years [7]. The literature lacks accurate data about the prevalence of distinct variants of $\mathrm{MO}$; however, neurogenic $\mathrm{MO}$ is often described as

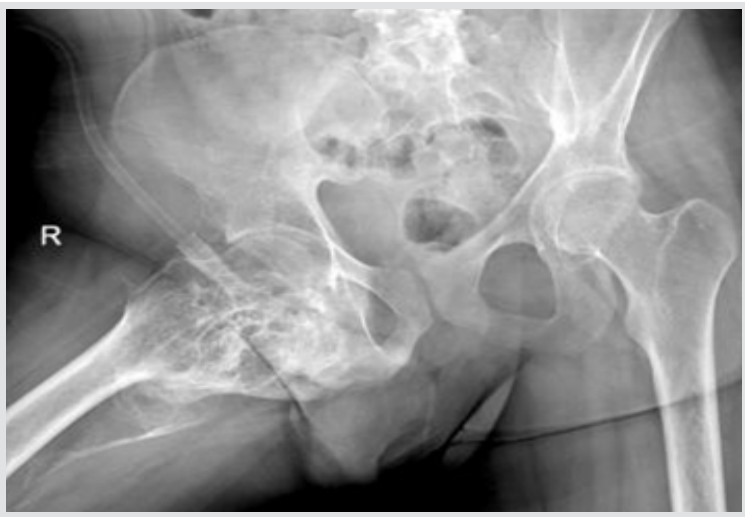

Figure 9: Case III;a radiographat presentation revealingossification around the righthip joint with fixed external rotation.

being a rare condition $[16,17]$. In our report, we described three cases of $\mathrm{MO}$ of the hip joint that was managed at our institute between the years 2016 and 2020. All three patients had an antecedent history of brain or spinal cord injury. The patient in Case II had also sustained a direct injury to the left hip joint that resulted in an acetabular fracture, which may have contributed to the pathophysiology of $\mathrm{HO}$ in this particular case. The involved site in our 3 cases is consistent with previous reports, suggesting that $\mathrm{MO}$ ossificans around the hip joint is more often seen with neurogenic causes such as spinal cord injuries and cerebral palsy $[16,18]$. This is in contrast to traumatic cases of $\mathrm{MO}$ that often involve the flexor muscles of the arm and extensor muscles of the thigh $[7,19]$.

MO can present with a wide variety of symptoms, with pain and joint stiffness being the classic ones [7]. The site of the lesion and proximity to joints as well as the stage of the disease process are the key factors dictating symptomatology [7]. Pain often subsides as the lesion matures, while a limited range of motion of the adjacent joint often progresses and a subset of patients develop frank ankylosis resulting in the significant functional limitation of the affected joint $[7,20]$. None of our patients complained of pain or evident swelling at the time of presentation; they all presented merely with a limited range of motion at the hip joint.
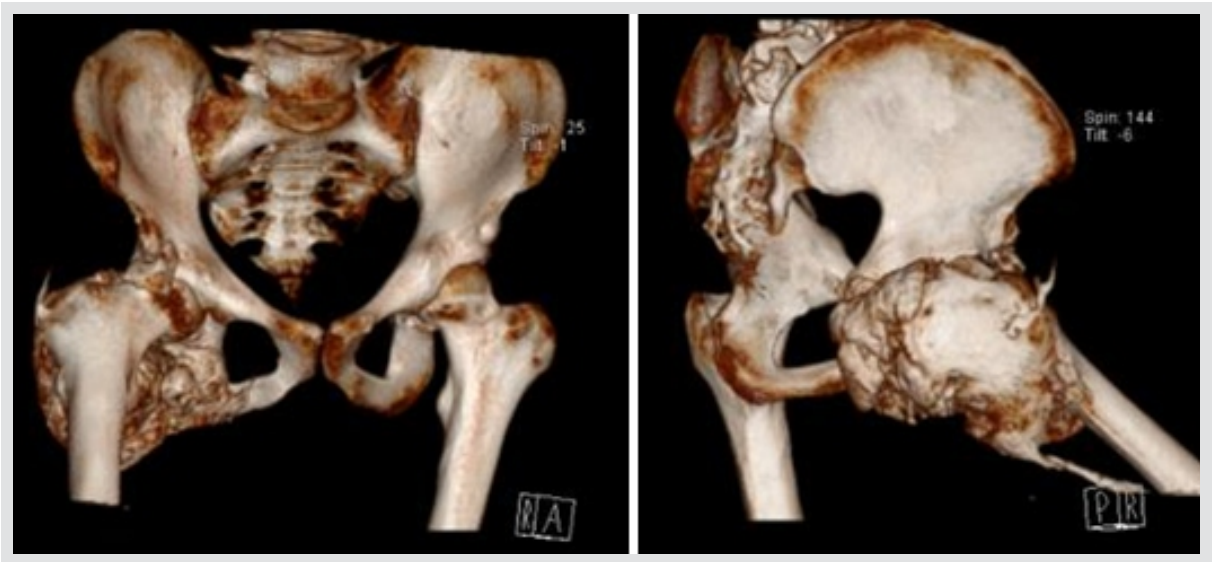

Figure 10: Case III; computerized tomography-3D showingheterotopic ossification surrounding the right hip joint and partial fusion of the proximal femur and dorsal acetabulum/ischium, causing fixed external rotation of the right femur.

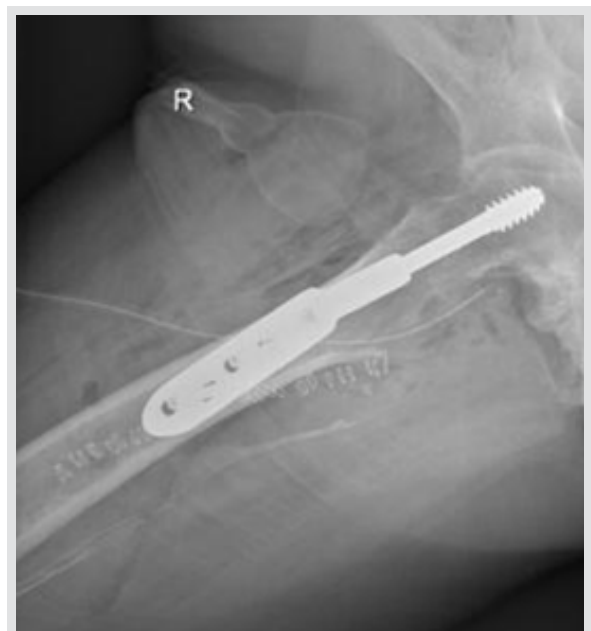

Figure 11: Case III;radiograph post excision of heterotopic ossification and dynamic hip screw insertion. 
Imaging studies constitute a fundamental part in the diagnostic process of MO [7]. Radiographic findings in many cases are sufficient to establish the diagnosis; however, more sophisticated imaging modalities, such as CT scan, MRI, or bone scintigraphy, may be necessary to reach a final diagnosis $[7,8,9,10,11]$. CT scans are superior to radiographs as they allow an accurate assessment of the lesion pre-operatively and they best delineate the pattern of calcification [6,21].For our patients, we ordered CT scan of the pelvis with 3D reconstruction to check the site and distribution of the $\mathrm{HO}$ as well as determine its relationship to the joint as either attached or separated by soft tissue. A bone scan can reveal increased uptake in the involved area and is considered very sensitive if ordered early enough in the disease course; however, literature lacks clear evidence regarding the practical value that bone scintigraphy offers in determining the appropriate timing for surgical intervention $[7,11]$. Historically, it has been suggested that SAP in the setting of traumatic MO may start to increase 3 weeks after the initial injury and levels peak, and can reach values that exceed 10 times the normal limit, at approximately 10 weeks and then return to normal levels by 18 weeks $[7,20,22]$. However, SAP level merely cannot determine the maturity or activity of a lesion as it can remain normal even in active lesions [22]. Thus, SAP levels need to be interpreted within both the clinical and radiographic contexts. Upon presentation, all our cases had SAP levels within normal limits.

Since MO ossificans is generally a self-limiting pathology, surgical excision is not the primary choice of treatment in most cases [3, 23]. Failure of non-surgical approach in controlling the symptoms, the presence of intractable pain, neurovascular compromise caused by compression effect, or decreased range of motion affecting the quality of life is all valid indications for surgical management of MO $[7,13,17]$. When surgery is indicated, excision of the lesion is performed after the lesion has matured; a decision best made with input from the clinical, laboratory (i.e., SAP level), and radiological aspects $[17,19]$. In the three cases presented here, the indication for surgical management was the significant reduction in the range of motion around the affected hip joint(s),causing severe limitation of activities of daily living. Based on the findings of the different modalities of imaging that were ordered and the SAP levels, all the cases were considered to be mature MO before surgery. Patients were consented for arthrolysis versus total hip replacement. In the first case, the patient was young and had bilateral hip involvement. In addition, his CT scan revealed the presence of soft tissue separating the ossification from the actual femur bone around the hip joint. This patient successfully underwent staged surgical excision of the ossifications, one side at a time. In the second case, arthrolysis was not possible intraoperatively due to the extensive connection between the $\mathrm{HO}$ and the hip joint. The patient thus had a total hip replacement. The third patient underwent excision of the ossification but sustained a stable intertrochanteric fracture intraoperatively, for which a DHS was inserted.

Previous reports suggest that post-operative physiotherapy and short-term indomethacin can both contribute to improved outcomes and decreased risk of recurrence [24, 25]. Some researchers also suggest that delivery of radiation locally, whether postoperatively following excision or as part of non-surgical approaches, was associated with positive outcomes [17, 19, 25]. Unfortunately, this modality is not available at our institute. Our three patients all received physiotherapy and oral indomethacin following. At most recent follow-up visits, all three patients demonstrated substantial improvement in the range of motion around the affected hip joint. Patients I and II became able to walk independently and perform all activities of daily living with no limitations. Moreover, they did not demonstrate any signs of recurrence of HOs, whether clinically or radiographically. Unfortunately, our last patient died 1 month after surgery due to pulmonary embolism, making follow-ups for post-operative outcome assessment not possible.

\section{Conclusion}

Although MO without a history of direct trauma is considered to be rare, this condition needs to be addressed in more detail in future studies as incidence seems to be increasing with correlation to road traffic accidents yielding brain/spinal cord injuries. Neurogenic MO of the hip joint introduces significant limitation in the activities of daily living, and thus, the quality of life. This condition must be addressed aggressively and managed surgically when indicated. The exact operative approach and timing of surgery are best determined by clinical, laboratory, and radiological inputs. CT scans with $3 \mathrm{D}$ reconstruction provide valuable data in determining the best surgical approach. Bone scans and SAP levels can provide useful input regarding the maturity of the lesion. For MO of the hip joint, surgical management by arthrolysis or total hip replacement, followed by post-operative physiotherapy and oral indomethacin, seems to provide satisfactory results.

\section{Clinical Message}

Although rare, the neurogenic MO of the hip needs clinical evaluation of its maturity through bone scan and alkaline phosphatase, followed by CT-3D evaluation and surgical planning. Arthrolysis or hip replacement showed significant improvement in range of motion and daily activity when associated with physiotherapy and indomethacin. 
1. Gindele A, Schwamborn D, Tsironis K, Benz-Bohm G. Myositis ossificans traumatica in young children: Report of three cases and review of the literature. PediatrRadiol 2000;30:451-9.

2. Sharma A, Behar M. Heterotopic ossification in fibrodysplasia ossificans progressiva. J Pediatr 2015;166:204.

3. Kransdorf MJ, Meis JM, Jelinek JS. Myositis ossificans: MR appearance with radiologic-pathologic correlation. AJR Am J Roentgenol 1991;157:1243-8.

4. Goyal K, Pettis CR, Bancroft AE, Wasyliw CW, Scherer KF. Myositis ossificans in the thigh of a lacrosse player. Orthopedics 2015;38:468, 515-518.

5. Huang H, Cheng WX, Hu YP, Chen JH, Zheng ZT, Zhang P. Relationship between heterotopic ossification and traumatic brain injury: Why severe traumatic brain injury increases the risk of heterotopic ossification. J OrthopTranslat 2018;12:16-25.

6. Shore EM, Xu M, Feldman GJ, Fenstermacher DA, Cho TJ, Choi $\mathrm{IH}$, et al. A recurrent mutation in the BMP type I receptor ACVR1 causes inherited and sporadic fibrodysplasia ossificans progressiva. Nat Genet 2006;38:525-7.

7. Walczak BE, Johnson CN, Howe BM. Myositis ossificans. J Am AcadOrthopSurg 2015;23:612-22.

8. Kaplan FS, Glaser DL, Hebela N, Shore EM. Heterotopic ossification.J Am AcadOrthopSurg 2004;12:116-25.

9. Papp DF, Khanna AJ, McCarthy EF, Carrino JA, Farber AJ, Frassica FJ. Magnetic resonance imaging of soft-tissue tumors: Determinate and indeterminate lesions. J Bone Joint Surg Am 2007;89Suppl 3:103-15.

10. Shehab D, Elgazzar AH, Collier BD. Heterotopic ossification.J Nucl Med 2002;43:346-53.

11. Tyler P, Saifuddin A. The imaging of myositis ossificans. SeminMusculoskeletRadiol2010;14:201-16.

12. Lipscomb AB, Thomas ED, Johnston RK. Treatment of myositis ossificans traumatica in athletes. Am J Sports Med 1976;4:111-20.

13. Ellerin BE, Helfet D, Parikh S, Hotchkiss RN, Levin N, Nisce $\mathrm{L}$, et al. Current therapy in the management of heterotopic ossification of the elbow: A review with case studies. Am J Phys Med Rehabil 1999;78:259-71.
14. Kan L, Kessler JA. Evaluation of the cellular origins of heterotopic ossification. Orthopedics 2014;37:329-40.

15. Medici D, Olsen BR. The role of endothelial-mesenchymal transition in heterotopic ossification. J Bone Miner Res 2012;27:1619-22.

16. Law-Ye B, Hangard C, Felter A, Safa D, Denormandie P, Genet F, et al. Pre-surgical CT-assessment of neurogenic myositis ossificans of the hip and risk factors of recurrence: A series of 101 consecutive patients. BMC MusculoskeletDisord 2016;17:433.

17. Oc Y, Ozcan MS, Sezer HB, Kilinc BE, Eren OT. Nontraumatic myositis ossificans of hip: A case presentation. Case Rep Orthop 2016;2016:1982656.

18. Juneja M, Jain R, Mishra D, Gautam VK. Myositis ossificans of bilateral hip joints in a patient with diplegic cerebral palsy.J ClinNeurosci 2011;18:580-1.

19. Mavrogenis AF, Soucacos PN, Papagelopoulos PJ. Heterotopic ossification revisited. Orthopedics 2011;34:177.

20. Garland DE. A clinical perspective on common forms of acquired heterotopic ossification. ClinOrthopRelat Res 1991;263:13-29.

21. Zeanah WR, Hudson TM. Myositis ossificans: Radiologic evaluation of two cases with diagnostic computed tomograms. ClinOrthopRelat Res 1982;168:187-91.

22. Orzel JA, Rudd TG. Heterotopic bone formation: Clinical, laboratory, and imaging correlation. J Nucl Med 1985;26:125-32.

23. Aneiros-Fernandez J, Caba-Molina M, Arias-Santiago S, O’Valle F, Hernandez-Cortes P, Aneiros-Cachaza J. Myositis ossificans circumscripta without history of trauma.J Clin Med Res 2010;2:142-4.

24. Spinzia A, Moscato G, Broccardo E, Castelletti L, Maglitto F, Orabona GD, et al. A rare isolated unilateral myositis ossificans traumatica of the lateral pterygoid muscle: A case report.J Med Case Rep 2014;8:230.

25. Benetos IS, Mavrogenis AF, Themistocleous GS, Kanellopoulos AD, Papagelopoulos PJ, Soucacos PN. Optimal treatment of fibrodysplasia ossificans progressiva with surgical excision of heterotopic bone, indomethacin, and irradiation.J SurgOrthopAdv 2006;15:99-104. 


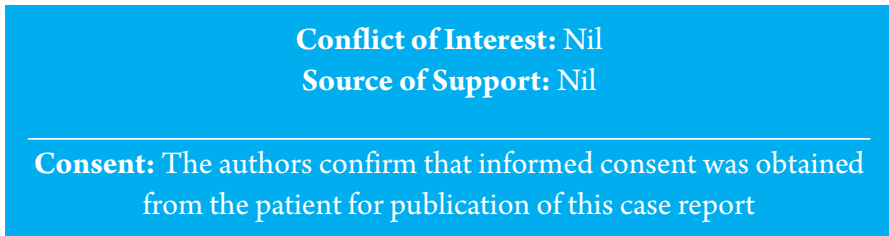

Conflict of Interest: Nil

Source of Support: Nil

from the patient for publication of this case report

\section{How to Cite this Article}

Hammad Y, Akiely R, Hajjaj N, Tahboub F, Al-Ajlouni J. The surgical management of the rare neurogenic myositis ossificans of the hip: A report of 3 cases. Journal of Orthopaedic Case Reports 2021 March;11(3): 45-51. 